\author{
UNIVERSIDADE DE SÃO PAULO \\ INSTITUTO DE RELAÇÕES INTERNACIONAIS
}

JAMESON VINÍCIUS MARTINS DA SILVA

\begin{abstract}
A TENSÃO ENTRE O NACIONAL E O LOCAL NO ÂMBITO DAS POLÍTICAS MIGRATÓRIAS: O ACESSO DOS MIGRANTES AOS SERVIÇOS DE SAÚDE NAS CIDADES DE SÃO PAULO E BARCELONA
\end{abstract}

SÃO PAULO 


\title{
A TENSÃO ENTRE O NACIONAL E O LOCAL NO ÂMBITO DAS POLÍTICAS MIGRATÓRIAS: O ACESSO DOS MIGRANTES AOS SERVIÇOS DE SAÚDE NAS CIDADES DE SÃO PAULO E BARCELONA
}

\author{
Dissertação apresentada ao Programa de Pós- \\ Graduação em Relações Internacionais do Instituto \\ de Relações Internacionais da Universidade de São \\ Paulo, para obtenção do título de Mestre em Ciências \\ Orientadora: Prof. Deisy de Freitas Lima Ventura
}

Versão corrigida

A versão original se encontra disponível na Biblioteca do Instituto de Relações Internacionais

SÃO PAULO 
Autorizo a reprodução e divulgação total ou parcial deste trabalho, por qualquer meio convencional ou eletrônico, para fins de estudo e pesquisa, desde que citada a fonte.

Ficha catalográfica

Biblioteca do Instituto de Relações Internacionais

Silva, Jameson Vinícius Martins da.

A tensão entre o nacional e o local no âmbito das políticas migratórias: o acesso dos migrantes aos serviços de saúde nas cidades de São Paulo e Barcelona / Jameson Vinícius Martins da Silva -- Orientadora: Deisy de Freitas Lima Ventura. São Paulo: 2017. 82 p.

Dissertação (Mestrado) - Instituto de Relações Internacionais. Universidade de São Paulo.

1. Migração. 2. Cidades. 3. Política de Migração. 4. Política de Saúde. I. Ventura, Deisy de Freitas Lima. II. Título. 


\section{RESUMO}

O teor securitário que compromete a eficiência das políticas migratórias na esfera nacional, a distribuição de competências entre os diversos níveis de governo e a proximidade entre as autoridades locais e os cidadãos fazem das cidades uma importante unidade de análise para a compreensão do fenômeno migratório. A presente dissertação busca verificar de que forma as cidades de São Paulo, no Brasil, e Barcelona, na Espanha, respondem às lacunas das respectivas políticas migratórias nacionais, examinando particularmente o acesso dos migrantes aos serviços de saúde na dimensão local. Ao reconhecer que a interface entre a mobilidade e a saúde humanas tende a expandir-se, eis que tanto as políticas migratórias como a sua ausência causam impacto significativo sobre a saúde dos migrantes e das sociedades de acolhida ou trânsito, a pesquisa investiga o papel das políticas migratórias na efetivação dos direitos humanos dos migrantes, em especial o direito à saúde. Determinadas iniciativas de administrações locais tentam responder às demandas geradas pelo fenômeno migratório, e se mostram comprometidas com uma agenda de proteção dos direitos dessas populações, em claro contraste com a orientação da legislação e da política migratória do plano nacional. Como já demonstra a literatura sobre migrações e governos locais, as cidades de São Paulo e de Barcelona demonstram tal empenho, embora suas respectivas administrações atuem em contextos jurídico-institucionais distintos: um de escassa consistência jurídica, no caso brasileiro, e outro de extensa legislação de teor restritivo, no caso espanhol.

Palavras-chave: políticas migratórias, cidades, direito à saúde, São Paulo, Barcelona

\section{ABSTRACT}

The securitarian tone hindering the efficiency of migration policies in the national realm, the distribution of competences among several government levels and the closeness between local authorities and citizens, all make cities an important unit of analysis for understanding the migration phenomenon. This Master's thesis aims to assess how the cities of Sao Paulo, in Brazil, and Barcelona, in Spain, respond to the gaps of their respective national migration policies, examining particularly the access of migrants to healthcare services at the local level. Admitting that the interplay between human mobility and health tends to expand, since both migration policies and their absence cause a significant impact on the health of migrants and reception and transit societies, this research explores the role of migration policies in making migrants' human rights effective, notably the right to health. Some initiatives of local administration seek to meet the demands sparked by migration and seem committed to a protective agenda of these population's rights, in a clear-cut contrast with the orientation of national migration laws and policies. As the literature on migration and local government exposes, the cities of Sao Paulo and Barcelona display such an effort, although their respective governments work in distinctive legal and institutional contexts: one of scarce legal consistency, in the Brazilian case, and another in an extensive legislation of restrictive contents, in the Spanish case.

Key words: migration policies, cities, right to health, Sao Paulo, Barcelona 


\section{INTRODUÇÃO}

Nas últimas décadas, as migrações internacionais atingiram dimensões absolutas inéditas. O número oficial de migrantes internacionais saltou de 77 milhões em 1975 para 244 milhões em 2015 (ONU, 2016). Embora a cifra corresponda em termos relativos a 3,3\% da população mundial, menor que os $5 \%$ do início do século passado, qualitativamente os fluxos se diversificaram e concernem hoje praticamente todas as regiões do planeta, seja pela partida, pela acolhida, pelo trânsito ou pelos três ao mesmo tempo. Regiões antigamente de partida -- sobretudo o continente europeu -- tornaram-se regiões de recepção dos migrantes, e novas zonas de atração emergiram, particularmente no Sul global, como o Golfo Pérsico, os BRICS e algumas partes da África (WIHTOL DE WENDEN, 2013).

Se adicionamos às migrações internacionais os migrantes internos aos Estados, estimados em 740 milhões (PNUD, 2009), constatamos que o fenômeno das migrações se tornou incontornável no mundo contemporâneo e adquiriu, portanto, relevância crescente tanto em termos das políticas estatais que buscam gerir esses fluxos quanto nas relações internacionais. A despeito da abrangência e do volume das migrações atuais, até muito recentemente o campo de estudos das relações internacionais não dedicou, entre suas abordagens mais tradicionais centradas nos Estados, um espaço à altura da repercussão gerada pela mobilidade humana (MOULIN, 2010, p. 9).

Ainda assim, no plano global, desde o início dos anos 2000, houve uma profusão de estudos e iniciativas por parte das organizações internacionais no sentido de incrementar a cooperação internacional em matéria de migrações, que "repentinamente estava por toda parte, no sistema onusiano e além dele" (NEWLAND, 2005). Tais iniciativas teriam constituído uma incipiente governança global das migrações, à qual se dirigem muitas críticas, entre elas, a de que sua agenda seria elitista e verticalizada, posto que seus principais implicados, os Estados, tanto geradores como receptores de mão-de-obra, sustentam uma visão utilitária e desenvolvimentista do que se chama "gestão dos fluxos", isto é, tendem a reduzir a questão migratória ao incremento dos ganhos econômicos derivados da mão-de-obra migrante (PÉCOUD, 2014; BARALDI, 2014).

No entanto, mesmo que reconheçam a importância das migrações de um ponto de vista estritamente econômico, observam-se entre os Estados diferentes padrões de controle de fronteiras. No sentido Sul-Norte, o direito de saída do Sul é relativamente livre, enquanto a entrada no Norte 
é restrita, particularmente à mão-de-obra pouco qualificada; entre os países do Norte, o trânsito e a salvaguarda de direitos são mais homogêneos, gerando fluidez migratória e riqueza; do Sul ao Sul, a entrada e a saída são grosso modo abertas, sobretudo face a demandas de mão-de-obra, mas os direitos dos migrantes extremamente reduzidos; e por fim, no sentido Norte-Sul, embora o trânsito seja relativamente desobstruído, certos direitos como acesso à nacionalidade e à propriedade são bastante restritos (WIHTOL DE WENDEN, 2013).

Baseados no princípio da soberania sobre o controle das fronteiras, os Estados de recepção, particularmente os desenvolvidos, investem pesadamente em mecanismos de dissuasão e contenção dos fluxos migratórios em sua direção. Dessa forma, criam em torno de suas fronteiras estruturas espetaculares de segurança, motivados também por questões econômicas, em especial a opulência do mercado de segurança; e político-ideológicas, sobretudo os discursos xenófobos legitimados na disputa política interna que equiparam a presença dos migrantes à uma suposta ameaça às identidades locais e ao bem-estar público (RODIER, 2012). À postura intransigente das políticas desses Estados se podem atribuir as dezenas de milhares de mortes -- estimadas em ao menos 60 mil nas últimas duas décadas -- nas linhas de fronteira entre as zonas de atração e repulsão de migrantes (OIM, 2016). 


\section{CONSIDERAÇÕES FINAIS}

O estudo dos dois casos desenvolvido ao longo deste trabalho são exemplos de como os governos municipais ou subnacionais podem, dentro de seus limites de competências, responder à realidade migratória que lhes afeta diretamente, por vezes em oposição às decisões políticas tomadas no plano dos governos nacionais. Isto pode se dar porque, da perspectiva local, distinguem-se mais claramente as condições de vida concretas da população migrante, em contraponto à distante burocracia do alto escalão estatal. A eficiência desta resposta está sujeita a uma série de contingências de ordem política, jurídica ou econômica.

Em termos de suas relações com os governos nacionais no âmbito das políticas migratórias e de saúde para migrantes, utilizando a tipologia proposta por Scholten e Penninx (2016), podemos identificar distintos padrões de governança entre São Paulo e Barcelona. A primeira demonstrou algum investimento em uma governança multinível. A Prefeitura contou, por exemplo, com o acordo pontual com o governo federal para colocar em marcha o Centro de Referência e Acolhida de Imigrantes (CRAI), via Ministério da Justiça e o Termo de Cooperação 92 estabelecido com a Organização Pan-Americana da Saúde (OPAS/OMS), que viabilizou a implementação de uma série de medidas em relação aos programas de saúde pública dedicados à população migrante, com intermédio formal do Ministério da Saúde.

Ainda assim, não se pode exagerar a densidade da coordenação entre o nível local e nacional, dado o contexto de uma legislação nacional ainda extremamente desfavorável a uma agenda progressista de direitos aos migrantes e o atraso para uma atualização legislativa nessa direção. Na verdade, o vácuo jurídico em relação ao papel dos governos locais e a incongruência do Estatuto do Estrangeiro com os preceitos da ordem constitucional podem ter precipitado ao mesmo tempo uma orientação localista, ainda nos termos de Scholten e Penninx, no sentido de que a cidade, principal pólo migratório do Brasil, protagonizou a formulação e a implementação de políticas próprias para migrantes, de forma inédita no país.

A cidade de Barcelona, por sua vez, parece ter mantido ao longo dos últimos anos um padrão de governança mais próximo do que Scholten e Penninx denominariam "desacoplado" em relação ao governo nacional espanhol, e ao mesmo tempo multinível, com as OSCs e com as redes de cidades europeias. A ausência de coordenação política declarada entre os representantes dos governos municipal e autonômico e o governo de Madri no que concerne o trato das migrações na cidade já sugeria o "desacoplamento", porém ele se evidenciou complemento pelos desdobramento 
políticos da interação entre os diferentes níveis de governo em 2017. No escopo deste trabalho, a aprovação da "Lei de Universalização da Assistência Sanitária" na Catalunha, precedida pela judicialização do RDL 16/2012, demonstra o conflito direto entre as orientações políticas dos governos nacional e regional e, por extensão, municipal, dado o papel relevante do governo de Barcelona no sentido da inclusão sanitária. Em um contexto mais amplo, a intervenção do governo espanhol na Catalunha, em resposta à tentativa de independência oficial da região, no fim de 2017 , realça ainda mais a natureza historicamente conflitiva da relação entre os catalães e Madri.

Em ambas as cidades, podemos identificar o elemento comum da governança no sentido horizontal, uma vez que ambas as administrações dispuseram de uma intensa interação com as OSCs, cuja atuação se ampliou da pressão exercida cotidianamente pelo aperfeiçoamento e adaptação de serviços públicos e de sua implicação direta no fornecimento dos serviços, para a participação direta na formulação de textos legais e iniciativas públicas favoráveis à proteção dos direitos dos migrantes. A dimensão e o vulto de ambas as cidades no que se refere à incorporação de populações consideráveis de diferentes origens permitiu o que Nicholls e Uitermark (2016) identificam como economias de escala e escopo, que se traduziu em uma ampla gama de instituições que adensam os discursos e a visibilidade em relação à migração.

Em São Paulo, a presença de importantes comunidades, especialmente sul-americanas, de fluxos mais recentes, impulsionou a entrada do tema na agenda política da cidade e um processo altamente criativo de formulação de políticas públicas voltadas a essa parcela da população. Além disso, o governo municipal convocou, em diversas ocasiões, a participação da rede de organizações não-governamentais envolvida diretamente nas questões dos migrantes na cidade. Destacam-se, entre esses momentos de participação da sociedade civil, o Comitê Intersetorial da Política Municipal para Imigrantes, que redigiu a minuta da Lei Municipal para a População Imigrante e o Grupo de Trabalho (GT) formado no âmbito da SMS, que direcionou as atividades do TC 92 com a OPAS/OMS. 
Em Barcelona, com uma trajetória mais longa de recepção de migrantes, a extensa rede de apoio e representação de migrantes é tida, pelos próprios formuladores de políticas do âmbito municipal e autonômico, como parte essencial da política municipal. Essa participação do amplo tecido associativo da cidade contribui para o que Zapata-Barrero (2017) distingue como a formação de uma cultura pública comum baseada na diversidade característica da chamada abordagem intercultural para a integração de migrantes na cidade. No campo da saúde pública, a exclusão sanitária imposta pelo RDL 16/2012 e suas consequências negativas no nível local foram intensamente combatidas pelas OSCs defensoras do sistema de saúde de caráter universal, que chegaram também a subsidiar tanto o texto das "Instruccions" do SCS como o processo legislativo que culminou na Lei de Universalização da Assistência Sanitária.

É importante assinalar, contudo, que a atuação mais assertiva dos governos locais em matéria de migrações não suplanta a relevância de políticas nacionais e supranacionais coerentes com as garantias de direitos humanos. Pouco vale que alguns poucos focos no território se tornem oásis de um bem-estar mínimo aos migrantes se em poucos quilômetros de distância eles podem ter seus direitos fragilizados pela ausência dessa garantia. Daí que as respostas locais devem vir articuladas a uma política migratória mais ampla de garantia de direitos e provisão de recursos de toda ordem para que as políticas públicas funcionem efetivamente na ponta da cadeia.

Dentro deste debate, as políticas econômicas de austeridade vão claramente na contramão dessa necessidade, já que em lugar de ampliar a oferta de recursos e aprimorar políticas públicas, elas desmontam as bases dessas últimas, afetando invariavelmente os mais vulneráveis, entre eles, migrantes em condições de vida mais frágeis. Tanto o caso espanhol como o brasileiro, apesar dos últimos avanços legislativos específicos em matéria migratória, evidenciam essa incongruência. Ainda é escassa a avaliação dos impactos diretos das medidas de austeridade sobre as condições de vida da população migrante no Brasil e na Espanha, embora os primeiros estudos já sugiram uma deterioração da qualidade da atenção sanitária neste último. A evidência reunida sobre outros casos, como mencionado anteriormente, demonstra um dano inequívoco às condições de saúde das populações mais frágeis, entre as quais os migrantes de baixa renda.

Os casos de São Paulo e de Barcelona inserem-se em um contexto amplo em que a mobilidade humana alcançou patamares sem precedentes em escala global, de variadas motivações e em múltiplas direções, em que pesem as restrições ao (ou as omissões estatais diante do) ato de migrar. Na qualidade de cidades globais, é de se esperar que os seus governos empreendam 
políticas que estejam à altura da complexidade do fenômeno migratório, no sentido de convertêlo em fonte de bem-estar social, baseado na proteção dos direitos das pessoas que nelas residem. Se não o fazem, estas pessoas são relegadas à margem do corpo social urbano. É justamente a esta margem que em geral se atribuem as disfunções de um sistema econômico por sua própria dinâmica já profundamente desigual, transformando os migrantes em "bodes expiatórios" das crises econômicas e do mal-estar social.

Para além dos benefícios econômicos da mobilidade que as próprias organizações internacionais há tempos reconhecem como manifestos, a integração política e social de migrantes nas cidades - e idealmente em todas as escalas geográficas - representaria um avanço civilizatório do reconhecimento da condição humana em todos os seus aspectos. O direito à saúde examinado por este trabalho na escala local, porém já consagrado em acordos internacionais, constitui um dos aspectos mais basilares de nossa condição como pessoas, o que nos impele a almejar sua proteção o mais horizontal e indiscriminadamente possível. Esta proteção se materializa na escala das cidades, o que sugere que delas pode emergir uma nova concepção do que consideramos ser cidadão. 


\section{REFERÊNCIAS BIBLIOGRÁFICAS}

AGUIAR, B.; NEVES, H.; LIRA, M. Alguns aspectos da saúde de imigrantes e refugiados recentes no município de São Paulo. Boletim CEInfo Análise, São Paulo, ano X, n. 13, 2015.

AGUIAR, M.; MOTA, A. O Programa Saúde da Família no bairro do Bom Retiro, SP, Brasil: a comunicação entre bolivianos e trabalhadores de saúde. Interface, v. 18, n. 50, pp. 493506, 2014.

AJUNTAMENT DE BARCELONA. La població estrangera de Barcelona, informes estadístics, Gener 2017. Disponível em:

< http://www.bcn.cat/estadistica/catala/dades/inf/pobest/pobest17/pobest17.pdf >. Acesso em: 20 Jun. 2017

Pla de Treball d'Immigració 2012-2015. Disponível em

<http://www.bcn.cat/novaciutadania/arees/ca/acollida/plans.html. Acesso em out. 2014.> Acesso em 03 Out. 2014

Pla Barcelona Interculturalitat., 2010. Disponível em $<$ http://www.bcn.cat/novaciutadania/pdf/ca/dialeg/programes/Pla_BCN_Interculturalitat_ca.pdf. $>$ Acesso em 03 Out. 2014

La salut de la població immigrant de Barcelona. Disponível em: <http://www.bcn.cat/novaciutadania/pdf/ca/salut/estudis/salut_immigrants_ASPB_BCN_ca.pdf $>$ . Acesso em: 20 Mai 2016.

Carta de la Alcaldesa Ada Colau i Ballano al Presidente del Gobierno

Mariano Rajoy, a 5 de septiembre de 2015. Disponível em $<$ http://ajuntament.barcelona.cat/premsa/wp-

content/uploads/2015/09/CartaRajoyCiutatRefugi_DEF.pdf> Acesso em 30.07.2017

AMRITH, Megha. Pathways to urban citizenship for low-income migrants in São Paulo. Citizenship Studies, United Nations University on Globalization, Culture and Mobility, Barcelona, 2015.

Cities as Humanitarian Actors in Contexts of Displacement. Policy Report n. 04/02. United Nations University on Globalization, Culture and Mobility, Barcelona, 2017.

BAENINGER, R. São Paulo e suas migrações no final do século 20. São Paulo em Perspectiva, v. 19, n. 3, pp. 84-96, 2005.

BARALDI, Camila Bibiana Freitas. 2014. 151f. Migrações internacionais, direitos humanos e cidadania sul- americana: o prisma do Brasil e da integração sul-americana. Tese de doutorado, Instituto de Relações Internacionais, IRI-USP, São Paulo, 2014. 
BOSCH, X. et al. The painful effects of the financial crisis on Spanish health care. International Journal of Health Services; v. 44, pp. 25-51, 2014.

BOUCHER, Manuel. Les théories de l'intégration à l'épreuve de la régulation sociale. Vie Sociale, n. 2, pp.23-43, 2007.

BRASIL. 1988. Constituição da República Federativa do Brasil.

Presidência da República. Casa Civil. Subchefia para Assuntos Jurídicos. Lei $n^{\circ} 6.815$, de 19 de agosto de 1980. Define a situação jurídica do estrangeiro no Brasil, cria o Conselho Nacional de Imigração. Disponível em: <http://www.planalto.gov.br/ccivil_03/leis/L6815.htm>. Acesso em: 15 out. 2014.

BURÓN, Andrea. 2012. 308f. Barreras de acceso de la población inmigrante a los servicios de salud en Catalunya. Tesis doctoral, Universitat Autònoma de Barcelona (UAB), Bellaterra, 2012.

CATALUNYA. Llei 10/2010, del 7 de maig, d'acollida de les persones immigrades i de les retornades a Catalunya.

CORELLA, Ángeles Solanes. La realidad local de la inmigración. Cuadernos Electrónicos de la Filosofía del Derecho, n. 10, 2004.

CSDH (Comissão sobre os Determinantes Sociais da Saúde). Closing the gap in a generation: health equity through action on the social determinants of health. OMS, Genebra, 2008.

ESPAÑA. Ley Orgánica 4/2000, sobre derechos y libertades de los inmigrantes en España. . Ley Orgánica 2/2009, de reforma de la LO 4/2000.

FAUSER, M. Autoridades locales e integración política en ciudades de nueva inmigración. Los casos de Madrid y Barcelona, in: Zapata-Barrero, Ricard und Gemma Pinyol (ed.). Los gestores del proceso de inmigración. Actores y redes de actores en España y en Europa, Barcelona: Fundació CIDOB, pp. 131-148, 2008.

FRASER, Nancy. Rethinking the public sphere: a contribution to the critique of actually existing democracy. Social Text, v. 25, n. 26, pp. 26-80, 1990.

FREITAS, P. T. Migrações internacionais e cidadania local: um estudo sobre a formação de um novo domínio de agência na cidade de São Paulo. In: II Encontro Internacional Participação, Democracia e Políticas Públicas, Unicamp, Campinas, 2015.

FREITAS, P. T. Migrações internacionais e cidadania local: um estudo sobre a formação de um novo domínio de agência na cidade de São Paulo. In: BAENINGER et al (org.). Imigração haitiana no Brasil. 1 ed., p. 427-450, Paco Editorial, Jundiaí, 2016. 
GONZALEZ, Juan Miguel Rosa; ANEZ, Miguel Eduardo Moreno. Ascenção e queda do novo "Oásis Catalão" (1980-2010): uma perspectiva institucional da organização política da Catalunha. Rev. Psicologia Política, São Paulo, v. 11, n. 21, 2011.

IPPDH (Instituto de Políticas Públicas em Direitos Humanos do Mercosul). Migrantes regionais na cidade de São Paulo: direitos sociais e políticas públicas. Buenos Aires, 2017. Disponível em: <http://www.ippdh.mercosur.int/wp-content/uploads/2017/01/Migrantesregionales-en-San-Pablo_PT-1.pdf>. Acesso em: 02.01.2017

KEROUEDAN, Dominique. Comment la santé est devenue un enjeu géopolitique, Le Monde Diplomatique, pp. 16-17, Jul. 2013.

LLOP-GIRONÉS et al. Accesso a los servicios de salud de la población extranjera en España. Revista Española de Salud Pública, v. 88, n.6, pp. 715-734, 2014.

MARROW, H. B. Access not denied? The role American localities can play. Field Actions Science Reports, 2010. Disponível em <http://factsreports.revues.org/483 >. Acesso em 03 Abr. 2017.

MARX, Vanessa. 2008. Las ciudades como actores políticos en las relaciones internacionales. Tesis doctoral. Universidad Autónoma de Barcelona, Bellaterra, 2008.

MÉNDEZ, E.; SAGARRA, E. Droits des étrangers et politiques en matière de protection de la santé en Catalogne. Migrations Société, CIEMI, vol. 23, n. 134-135, pp. 215-226, 2011.

MILANI, Carlos. O princípio da participação social na gestão de políticas públicas locais: uma análise de experiências latino-americanas e europeias. Revista de Administração Pública, v. 42, n. 3, pp. 551-579, FGV, Rio de Janeiro, 2008

MOULIN, Carolina. Eppur si muove: Mobilidade Humana, Cidadania e Globalização. Contexto Internacional, v. 33, n. 1, pp. 47-69, 2011.

NEWLAND, Kathleen. The governance of international migration: mechanisms, processes and institutions. Global Commission on International Migration, 2005.

NICHOLLS, W; UITERMARK, J. Migrant cities: place, power, and voice in the era o super diversity. Journal of Ethnic and Migration Studies, v. 42, n. 6, pp. 877-892, 2016.

NUNES, João. Security, Emancipation and the Politics of Health. Routledge, 2014.

OIM, OMS, ACNUDH - Organização Internacional para as Migrações, Organização Mundial de Saúde, Alto Comissariado das Nações Unidas para os Direitos Humanos.

International migration, health and human rights. Geneva, 2013. Disponível em: <http://www.ohchr.org/Documents/Issues/Migration/WHO_IOM_UNOHCHRPublication.pdf>. Acesso em: 25 Fev. 2016. 
OIM (Organização Internacional para as Migrações). World Migration Report 2015: Migrants and Cities, New Partnerships to Manage Mobility, Genebra, 2015.

Fatal Journeys, Volume 2: Identification and Tracing of Dead and Missing Migrants. Genebra, 2016.

OMS (Organização Mundial da Saúde). Constitution of the World Health Organization, 1946. Disponível em:

<http://www.who.int/governance/eb/who_constitution_en.pdf.> Acesso em: 25. Jun. 2016.

ONU (Organização das Nações Unidas). International Migration Report 2015, Departament of Economic and Social Affairs, Population Division, 2016. Disponível em: < http://www.un.org/en/development/desa/population/migration/publications/migrationreport/docs/ MigrationReport2015.pdf>. Acesso em: 20 Jan. 2017

ONU-HABITAT, OMS. Hidden Cities: unmasking and overcoming health inequities in urban settings. Genebra, 2010.

OPAS/OMS (Organização Pan-Americana da Saúde). A implantação da política municipal de saúde para imigrantes e refugiados na cidade de São Paulo/SP. Inovação e Direito à Saúde na cidade de São Paulo (2013-2016), pp. 39-52, 2017. Disponível em < http://iris.paho.org/xmlui/bitstream/handle/123456789/34091/OPASBRA17011por.pdf? sequence=5\&isAllowed=y> . Acesso em 08 Jun. 2017.

OPAS/OMS; SMS. Relatório Técnico TC 92: Fortalecimento e qualificação da atenção básica e das redes de atenção à saúde no Município de São Paulo. 2017. Disponível em: < http://www.paho.org/bra/index.php?option=com_docman\&view=download\&slug=rt-tc-92a\&Itemid=965>. Acesso em: 14 Ago. 2017

PATARRA, N.; BAENINGER, R. Mobilidade espacial da população no Mercosul: metrópoles e fronteiras. Revista Brasileira de Ciências Sociais, v. 21, n. 60, pp. 83-102, 2006.

PASUCAT (Plataforma per una Atenció Sanitària Universal a Catalunya). Propostes per assolir SanitatxTothom a Catalunya, 2015. Disponível em: < https://drive.google.com/file/d/0B2opi6SzvyEKX3c1akRSVXBGelU/view>. Acesso em: 30 Set. 2016

PÉCOUD, Antoine. États-nations, mobilité et citoyenneté dans le discours international sur les migrations. Raisons politiques, v. 2, n. 54, pp. 67-85, 2014.

PERRUCHOUD, Richard; REDPATH-CROSS, Jillyanne, (ed.). Glossary on Migration, International Organization for Migration, 2nd. ed., Genebra, 2011. 
PNUD. Human Development Report 2009. Overcoming Barriers: Human Mobility and Development. Nova York, 2009.

PORTHÉ et al. Changes in access to health care for immigrants in Catalonia during the economic crisis: opinions of health professionals and immigrants users. Health Policy, 2016.

PREFEITURA DE SÃO PAUlO. Plano Municipal de Saúde 2014-2017. 3. ed. São Paulo: Secretaria Municipal da Saúde, Prefeitura de São Paulo, 2014. Disponível em: <http://www.prefeitura.sp.gov.br/cidade/secretarias/upload/saude/planomunicipaldesaudeterceira edicao.pdf>. Acesso em: 25 fev. 2016.

. Secretaria Municipal de Saúde. Conselho Municipal de Saúde. Ata da 198

Reunião Plenária Ordinária, 13 agosto 2015, 2015a. Disponível em: <http://www.prefeitura.sp.gov.br/cidade/secretarias/upload/saude/ATA198REUNIAOORDINA RIADOPLENODOCMS130815\%20.pdf>. Acesso em: 25 fev. 2016.

. Secretaria Municipal de Saúde. Conselho Municipal de Saúde. Ata da 199a Reunião Plenária Ordinária, 10 setembro 2015, 2015b. Disponível em:

<http://www.prefeitura.sp.gov.br/cidade/secretarias/upload/saude/ATA\%20 da\%20199\%20Reunião\%200rdinaria\%20do\%20CMSSP\%20de\%2010_09_2015_aprovada.pdf> Acesso em: 25 junho 2017.

. Programação Anual de Saúde 2016. 2. ed. São Paulo: Secretaria Municipal da Saúde, Prefeitura de São Paulo, 2016. Disponível em:

<http://www.prefeitura.sp.gov.br/cidade/secretarias/upload/saude/planoanualdesaude2016.pdf . Acesso em: 25 fev. 2016.

. Programação Anual de Saúde 2017. São Paulo: Secretaria Municipal da Saúde, Prefeitura de São Paulo, 2017. Disponível em: < http://www.prefeitura.sp.gov.br/cidade/secretarias/upload/saude/Programacao\%20Anual\%20de \%20Saude\%202017.pdf>. Acesso em: 25 junho 2017.

PREFEITURA DE SÃO PAULO, OIT (Organização Internacional do Trabalho). Coordenação de Políticas para Migrantes na cidade de São Paulo, 2016.

PRICE, J.; SPENCER, S. City-level responses to migrant families with restricted access to welfare benefits: a European pilot study. Center on Migration, Policy and Society (COMPAS), Oxford Universiy, Oxford, 2014.

RECHEL et al. Migration and health in an increasingly diverse Europe. The Lancet, n. 381, pp. 1235-45, 2013.

REDER (Rede de Denúncia e Resistência ao RDL 16/2012). Cinco mitos para cinco años de exclusión sanitária. 2017. Disponível em: <https://reder162012.org/images/Informe2015/Abril2017/Abril2017.pdf>. Acesso em: 05 Mai. 2017. 
REIS, R. R. A política do Brasil para as migrações internacionais. Contexto Internacional, v. 33, n. 1, pp. 47-69, 2011.

RIDGLEY, Jennifer. Cities of Refuge: Immigration enforcement, police, and the insurgent genealogies of citizenship in US sanctuary cities. Urban Geography, v. 29, n. 1, pp. 53-77, 2008.

RODIER, Claire. Xénophobies Business. À quoi servent les contrôles migratoires? Paris, La Découverte, 2012. [livro eletrônico]

SÃO PAULO COSMÓPOLIS. Imigrantes em São Paulo: diagnóstico do atendimento à população imigrante no município e perfil dos imigrantes usuários de serviços públicos. Deisy Ventura e Feliciano Sá Guimarães (coord.), São Paulo, IRI-USP, 2017. No prelo.

SASSEN, S. Territory, authority, rights: from medieval to global assemblages. 2. ed. Princeton/Oxford, Princeton University Press, 2006.

SCHULTZ, C. Migration, health and urbanization: interrelated challenges. Background Paper of the World Migration Report 2015. The Expert Council of German Foundations on Integration and Migration, Berlim, 2014.

SCHOLTEN, P.; PENNINX, R. The multilevel governance of migration and integration. In: GARCÉS-MASCAREÑAS, B.; PENNINX, R. Integration processes and policies in Europe. IMISCOE Research Series, 2016.

SOUZA, Beatriz. Pelos imigrantes: experiências com direitos humanos e imigração na Prefeitura de São Paulo (2013-2014). Aracê (Direitos Humanos em Revista), v. 2, n. 3, 2015.

SPENCER, Sarah. The Migration Debate, The Policy Press, University of Bristol, 2011 [livro eletrônico]

STUCKLER, David; BASU, Sanjay. The Body Economic: eight experiments in economic recovery, from Iceland to Greece. Penguin, 2014 [livro eletrônico]

SUMPTION, M. Giving cities and regions a voice in immigration policy: can national policies meet local demands? Migration Policy Institute, Washington, DC, 2014.

TRUMMER, Ursula, NOVAK-ZEZULA, Sonja. Cost analysis of health care provision for irregular migrants and EU citizens without insurance. Final Report, OIM-EquiHealth, Viena, 2016.

VENTURA, D. F. L. O direito de migrar na Constituição Federal: o anacronismo brasileiro em matéria de cidadania e de mobilidade humana. In: Clèmerson Merlin Clève; Alexandre Freire. (Org.). Direitos fundamentais e jurisdição constitucional : análise, crítica e contribuições. 1 ed. Revista dos Tribunais RT, São Paulo, v. 1, pp. 319-334, 2014. 

. Mobilidade humana e saúde global. Revista USP, n. 107, pp. 55-64, 2015.

WALDMAN, T. Movimentos migratórios sob a perspectiva do direito à saúde: imigrantes bolivianas em São Paulo. Revista e Direito Sanitário, São Paulo, v. 12, n.1, pp. 90-114, 2011.

XAVIER, I. R. 2010. 263f. Projeto migratório e espaço: os migrantes bolivianos na região metropolitana de São Paulo. Dissertação de mestrado, Instituto de Filosofia e Ciências Humanas da Universidade Estadual de Campinas (Unicamp), Campinas, 2010.

WITHOL DE WENDEN, C. Faut-il ouvrir les frontières?, $2^{\mathrm{a}}$ ed., Paris, Presses de Sciences Po, 2013.

ZAPATA-BARRERO, R. Catalan autonomy-building process in immigration policy: conceptual, institutional and normative dimensions", In: A. Gagnon and M. Keating (eds.) Political Autonomy and Divided Societies: Imagining Democratic Alternatives in Complex Settings. Gordonsville, Virginia: Palgrave/MacMillan Table of Contents and Introduction), pp. 220-23, 2012a.

La communauté politique en tant que fondement d'une théorie politique catalane de l'immigration: enjeux clés. In: Labelle, M, Couture, J., Remiggi, F.W. (eds.). La Communauté Politique en question. Regards croisés sur l'immigration, la citoyenneté, la diversité et le pouvoir. Québec, Presses de l’Université du Québec, 2012b.

$$
\text { Interculturalism in the post-multicultural debate: a defence. }
$$

Comparative Migration Studies, v. 5, n. 14, 2017. 